ben ausgeschlossen werden (vgl. auch Pressemitteilung des Komitees für Grundrechte und Demokratie vom 03.07.2008, abrufbar unter: www.grundrechtekomitee.de). 25 der in dieser Studie Untersuchten waren bis zum Abschluss der Gerichtsverfahren über ihr reguläres Haftende hinaus untergebracht, vier von ihnen länger als 1 1/2 Jahre. Mit ein wenig Haftentschädigung ist es da nicht getan. Es ist Zeit für ein Umdenken! Die Folgerung des CDU-Politikers Jürgen Gehb (lt. Frankfurter Rundschau v. 24.07.2008) aus der restriktiven Rechtsprechung, die „neuen Erkenntnisse“ aus dem $\$ 66 \mathrm{~b}$ StGB zu streichen, ist sicher nicht der richtige Weg, sie wird der realen Bedrohung durch einschlägige Rückfälle auch nicht gerecht.

Michael Alex, Lehrstubl für Kriminologie, Kriminalpolitik, Polizeiwissenschaft, (Prof. Dr. Thomas Feltes M.A.) Ruhr-Universität Bochum, 44780 Bochum, Tel. 0234/32-28245/-25244, E-Mail: thomas.feltes@rub.de
Literaturangaben:

Bock 2007: Bock, Michael: Das Elend der klinischen Kriminalprognose, StV $5,2007,269$

Boetticher et al. 2006: Boetticher, Axel; Kröber, Hans-Ludwig; Müller-Isberner, Rüdiger; Böhm, Klaus M.; Müller-Metz, Reinhard; Wolf, Thomas: Mindestanforderungen für Prognosegutachten, NStZ 2006, 537.

Dahle 2006: Dahle, Klaus-Peter: Grundlagen und Methoden der Kriminalprognose. In: Kröber/Dölling/Leygraf/Sass (Hrsg.): Handbuch der Forensischen Psychiatrie, Band 3, Darmstadt 2006, 1 ff.

Frommel 2004: Frommel, Monika: Lebenslange Verwahrung angeblich nicht therapierbarer und extrem gefährlicher Sexualstraftäter seit 1998 in Deutschland und der Schweiz, NK 3/2004, 86.

Jehle et al. 2003: Jehle, Jörg-Martin; Heinz, Wolfgang; Sutterer, Peter: Legalbewährung nach strafrechtlichen Sanktionen - eine kommentierte Rückfallstatistik, Bonn, Mönchengladbach 2003.

Rusche 2004: Rusche, Stefan: In Freiheit gefährlich? - Eine Untersuchung zu Häufigkeit und Gründen falscher Kriminalprognosen bei psychisch kranken Gewaltverbrechern, Regensburg 2004.

\section{Fußnoten:}

1 Nach Berichten in „Der Spiegel“ v. 20.10.2003 und „Die Welt" vom 23.10.2003 waren in Bayern 30 Anträge zurückgewiesen worden, nach Auskunft der Bayerischen Staatsregierung vom 22.10.2007 lediglich 21.

\title{
Sicherungsverwahrung gem. $\$ 7$ Abs. 2 JGG und der Präventionsgedanke im Strafrecht - kritische Betrachtung eines legislativen Kunstgriffs
}

\section{Nina Nestler und Christian Wolf}

\section{Einleitung}

Mit dem Gesetz zur Einführung der nachträglichen Sicherungsverwahrung bei Verurteilungen nach Jugendstrafrecht vom 8.7.2008 ${ }^{1}$ fügte der Gesetzgeber einem umfassenden Projekt zur Reformierung des Straf- und Maßregelrechts ein weiteres Puzzleteil hinzu. Bereits seit Längerem konnte der aufmerksame Beobachter den Eindruck gewinnen, das Strafrecht mutiere mehr und mehr von einem Recht repressiver Sanktionen zu einer Art universell einsetzbarem, präventiv-sicherheitsrechtlichem „Breitschwert“ des Gesetzgebers. ${ }^{2}$ Eine besondere Rolle scheint dabei die Maßregel der Sicherungsverwahrung zu spielen, welche in den vergangenen zehn Jahren bereits ein bemerkenswertes Revival erlebte. Gemäß $\$ 7$ Abs. 2 JGG n. F. darf nun auch für nach Jugendstrafrecht Verurteilte nachträglich die Unterbringung in der Sicherungsverwahrung angeordnet werden, sofern nach der Verhängung einer Jugendstrafe von mindestens sieben Jahren wegen eines Verbrechens gegen das Leben, die körperliche Unversehrtheit, die sexuelle Selbstbestimmung oder nach $\$ \mathbb{S} 251$, 252, 255 StGB, durch welches das Opfer seelisch oder körperlich schwer geschädigt worden ist, vor Ende des Vollzugs Umstände erkennbar werden, die auf eine erhebliche Gefährlichkeit des Täters für die Allgemeinheit hinweisen.

Die Neuregelung des $₫ 7$ Abs. 2 JGG bildet ein Beispiel populistischer Ad-hoc-Aktionen des Gesetzgebers, mit dem er auf Beunruhigungen oder Ängste in der Bevölkerung reagiert. Unter dem Deckmantel eines vermeintlich gesteigerten Sicherheitsbedürfnisses ${ }^{3}$, das zu einem wesentlichen Teil jedoch aus überzogener medialer Berichterstattung resultiert ${ }^{4}$, antwortet man durch derlei Kunstgriffe auf scheinbare Bedrohungen um Handlungsstärke und Durchsetzungsfähigkeit zu demonstrieren.

II. Die Sicherungsverwahrung für Jugendliche im kriminalpolitischen Kontext

\section{Sicherungsverwahrung in Deutschland}

a) Die Entwicklung des Rechts der Sicherungsverwabrung für Erwachsene

Überlegungen zu einer dauerhaft sicheren Verwahrung nicht besserungsfähiger Gewohnheitsverbrecher zum Schutz der Allgemeinheit finden sich schon bei Franz von Liszt, der als mögliche Wirkungen der Strafe die Besserung, Abschreckung und Unschädlichmachung identifizierte. Gegen unverbesserliche Gewohnheitsverbrecher müsse sich die Gesellschaft schützen, schrieb von Liszt, wobei - da „köpfen und hängen “ sowie deportieren nicht in Betracht komme - eben „nur die Einsperrung auf Lebenszeit, bzw. auf unbestimmte Zeit“ bleibe. ${ }^{5}$ Mit dem Gesetz gegen gefährliche Gewohnheitsverbrecher und über Maßregeln der Besserung und Sicherung wurde in Deutschland das Institut der Sicherungsverwahrung in $\ 42 \mathrm{e}$ RStGB mit Wirkung zum 1.4.1934 eingeführt. ${ }^{6}$

Nach dem 2. Weltkrieg fand die Vorschrift des $\mathbb{4} 42 \mathrm{e}$ StGB ohne größere Änderungen in das bundesdeutsche Strafgesetzbuch Eingang. Die Justiz begegnete der Sicherungsverwahrung jedoch unter Verweis auf den vorangegangenen Missbrauch der Maßregel durch das NS-Regime mit größter Zurückhaltung. Diesen Bedenken und 
Vorbehalten trug schließlich der Gesetzgeber im Wege des 1. Strafrechtsreformgesetzes im Jahr 1969 Rechnung, mittels dessen $\$ 42 \mathrm{e}$ StGB eine erste grundlegende Änderung erfuhr. Man hob die Voraussetzungen für die Anordnung der Sicherungsverwahrung an und bestimmte als maximale Verwahrdauer bei erstmaliger Verhängung der Maßregel eine Höchstgrenze von zehn Jahren; gegenüber Heranwachsenden schloss man die Sicherungsverwahrung explizit aus. Hierbei war es der Legislative ein besonderes Anliegen, das Rechtsinstitut als ultima ratio staatlicher Reaktionen im Strafrecht verstanden zu wissen. Das 2. Strafrechtsreformgesetz überführte dann noch im gleichen Jahr $\mathbb{S}$ 42e StGB mit Wirkung zum 1.10.1973 in $\$ 66$ StGB. ${ }^{7}$

Galt die Maßregel der Sicherungsverwahrung bis Mitte der neunziger Jahre des 20. Jahrhunderts schon als Auslaufmodell, wurde sie gegen Ende dieser Dekade wiederentdeckt und zum Objekt zahlreicher gesetzgeberischer Aktivitäten. Die Medien berichteten über Straftäter, welche trotz fortbestehender Gefährlichkeit nach Ablauf der zehnjährigen Höchstverweildauer aus der Sicherungsverwahrung entlassen werden mussten, sodann in Freiheit erneute Rückfälligkeit zeigten und schwerste Straftaten begingen. Unter dem Eindruck von Fällen schwerer Sexualstraftaten begann der Gesetzgeber ab dem Jahr 1998 damit, sukzessive das Recht der Sicherungsverwahrung zu reformieren und auszuweiten. Beobachten ließ sich eine regelrechte „Renaissance der Sicherungsverwahrung “8 ; die Rede war auch vom Einsetzen einer „Verschärfungsspirale“9 . Eine erste größere Änderung realisierte sich noch im Wahljahr 1998 mit dem Gesetz zur Bekämpfung von Sexualdelikten und anderen gefährlichen Straftaten (Sexualdeliktebekämpfungsgesetz - SexualdelBekG). ${ }^{10}$ Hierauf folgte 2002 die Einführung der vorbehaltenen Sicherungsverwahrung nach $\ 66$ a StGB ${ }^{11}$, bevor dann, als Reaktion auf die Entscheidung des Bundesverfassungsgerichts zu den Unterbringungsgesetzen der Länder Bayern und Sachsen-Anhalt im Jahr $2004^{12}$, die Legislative auf Bundesebene binnen kürzester Zeit mit $\$ 66 \mathrm{~b}$ StGB die gesetzlichen Voraussetzungen für die Anordnung einer nachträglichen Sicherungsverwahrung festlegte.

In einem für die bundesdeutsche Kriminalpolitik einmaligen Vorgang avancierte damit die Sicherungsverwahrung für Erwachsene mit ihren nunmehr drei Varianten, der im Urteil ausgesprochenen, der vorbehaltenen und der nachträglichen, binnen kürzester Zeit zu einem eigenen umfangreichen Problemkomplex. Selbst Befürworter der Regelungen beklagen die Unübersichtlichkeit des neuen Maßregelrechts, so u. a. „die Vielzahl unterschiedlicher Kriterien“ der $\int \mathbb{S} 66,66 \mathrm{a}, 66 \mathrm{~b} \mathrm{StGB} .{ }^{13}$

\section{b) Sicherungsverwahrung bei Jugendlichen und Heranwachsenden}

Als im Jahr 1953 Heranwachsende in den Geltungsbereich des Jugendgerichtsgesetzes einbezogen wurden, ermöglichte $\mathbb{} 106$ Abs. 2 JGG dem erkennenden Gericht, von einer Anordnung der Sicherungsverwahrung bei Heranwachsenden abzusehen. Wie sich jedoch zeigte, brachte man die Maßregel in der Praxis gegenüber 18- bis 21-Jährigen ohnehin kaum jemals zur Anwendung. ${ }^{14}$ Im Rahmen des 1. Strafrechtsreformgesetzes schloss der Gesetzgeber die Sicherungsverwahrung für Heranwachsende dann sogar explizit aus. Dabei trug man dem Umstand Rechnung, dass hinreichend verlässliche forensisch-psychiatrische Prognosen über das weitere Verhalten des Täters und dessen Gefährlichkeit aufgrund seines geringeren Lebensalters sowie des oftmals noch nicht abgeschlossenen Reifeprozesses kaum zu erstellen sind. ${ }^{15}$ Auch bei jungen Erwachsenen sah sich die höchstrichterliche Rechtsprechung immer wieder veranlasst, die Zurückhaltung beim Umgang mit der Maßregel zu betonen ${ }^{16}$, deren Anordnung stets einer ganz besonders sorgfältigen Prüfung bedür$\mathrm{fe}^{17}$. Gegenüber Jugendlichen bzw. nach Jugendstrafrecht verurteilten Heranwachsenden konnte eine Sicherungsverwahrung bislang gem. \ 7 JGG a. F. überhaupt nicht angeordnet werden.

Zwischenzeitlich hat auf Seiten des Gesetzgebers offensichtlich ein Umdenken stattgefunden. Bereits mit dem Gesetz zur Änderung der Vorschriften über die Straftaten gegen die sexuelle Selbstbestimmung und zur Änderung anderer Vorschriften vom 27.12.2003 $3^{18}$ ermöglichte man mit $\$ 106$ Abs. 3 JGG unter engen Voraussetzungen den Vorbehalt der Sicherungsverwahrung bei nach Erwachsenenstrafrecht verurteilten Heranwachsenden. Im Jahr 2004 erfolgte dann mit dem Gesetz zur Einführung der nachträglichen Sicherungsverwahrung ${ }^{19}$ erneut eine Erweiterung des $\$ 106$ JGG. Fortan ließen \106 Abs. 5 u. 6 JGG die Anordnung der nachträglichen Sicherungsverwahrung auch gegenüber Heranwachsenden zu, bei deren Verurteilung Erwachsenenstrafrecht Anwendung fand. Dabei lehnte sich die Legislative bei der Ausgestaltung der Voraussetzungen an die Vorschrift des $\$ 66 \mathrm{~b}$ StGB an. Begründet wurde diese Kehrtwende mit dem Sicherheitsbedürfnis der Allgemeinheit, das auch gegenüber Heranwachsenden, bei denen sich die Gefährlichkeit erst im Laufe des Strafvollzugs herausstellte, nicht geringer wiege, als gegenüber denjenigen Heranwachsenden, die schon zur Zeit ihrer Verurteilung offensichtlich gefährlich waren und bei denen man die Sicherungsverwahrung deshalb bereits im ursprünglichen Urteil vorbehielt. Ebenso gelte dies, sofern im Maßregelvollzug trotz Fortfalls der psychischen Störung in qualifizierter Weise die Gefährlichkeit des betreffenden Heranwachsenden bestätigt werde. ${ }^{20}$

Sehr bald nach den beiden wegweisenden Entscheidungen des Bundesverfassungsgerichts zur Sicherungsverwahrung im Jahre $2004^{21}$ kamen Forderungen auf, nunmehr die vorbehaltlose Anordnung der Sicherungsverwahrung nach $\mathbb{} 66 \mathrm{StGB}$ für Heranwachsende gleichermaßen einzuführen. ${ }^{22}$ Die Möglichkeit, diese Maßregel unter den Voraussetzungen des $\$ 106$ Abs. 3 JGG vorzubehalten, sei aufgrund der hohen Anforderungen der Norm kaum von praktischer Relevanz. Mit Blick auf die Vorschrift des \$106 JGG a. F. bemängelte man, es sei nicht einsehbar, weshalb bei nach Erwachsenenstrafrecht verurteilten Heranwachsenden, nicht die Möglichkeit einer Anordnung der Sicherungsverwahrung bereits im Urteil bestehe; dies werde den Sicherheitsbedürfnissen der Bevölkerung nicht gerecht. $^{23}$

Im Jahr 2006 erfolgte ein Vorstoß des Freistaats Bayern im Bundesrat, die nachträgliche Sicherungsverwahrung auch für Jugendliche, d. h. Personen im Alter zwischen 14 und unter 18 Jahren, zu ermöglichen. Jüngste Erfahrungen hätten Bedarf aufgezeigt, die Bevölkerung vor Entlassungen gefährlicher jugendlicher Straftäter aus dem Vollzug zu schützen, weil dem Gewaltpotential einzelner weniger nach Jugendstrafrecht verurteilter Täter nicht mit den Einwirkungsmöglichkeiten des Jugendstrafrechts begegnet werden könne. ${ }^{24}$

Der Bundesgesetzgeber hat nunmehr mit dem am 8.7.2008 verabschiedeten Gesetz zur Einführung der nachträglichen Sicherungsverwahrung bei Verurteilungen nach Jugendstrafrecht dem Maßregelrecht wieder ein neues Puzzlestück hinzugefügt. War die Verhängung der Sicherungsverwahrung bei 14- bis 17-jährigen Straftätern bislang ausgeschlossen, so eröffnet $\mathbb{} 7$ Abs. 2 JGG n. F. nunmehr auch bei Jugendlichen und nach Jugendstrafrecht verurteilten Heranwachsenden die Möglichkeit, die Maßregel nachträglich anzuordnen. 


\section{Emotion statt Empirie}

Schon bei Einführung der nachträglichen Sicherungsverwahrung für Erwachsene monierten Fachwelt und Öffentlichkeit, der Gesetzgeber drehe mit „fast üblicher Hektik am Rad des Maßregelrechts“25, das Gesetz sei mit „heißer Nadel“ ${ }^{\text {26 }}$ gestrickt. Es wird im Weiteren zu zeigen sein, dass dies auch neuerlich auf das vorliegende Gesetz zutrifft.

\section{a) Emotionen schüren}

Die Debatte um angemessene Strafen sowie den Schutz der Allgemeinheit vor hochgefährlichen Straftätern findet mittlerweile in den Medien und der Gesellschaft einen breiten Widerhall, der wiederum von der Politik dankbar aufgegriffen wird. Öffentliche Diskurse über strafrechtliche Sanktionen, Sicherheit oder Kriminalität schüren Emotionen und bieten den politischen Kontrahenten Gelegenheit zu Mobilisierung bzw. Profilierung. „Die Verwandlung unlenksamer und unheilbarer Sorgen über die individuelle Sicherheit in das dringende Bedürfnis, tatsächliche oder potentielle Verbrecher zu bekämpfen und so die öffentliche Sicherheit zu verteidigen, ist ein wirksamer politischer Kunstgriff, der sich bei Wahlen merklich auszahlt. " 27 Politik und Massenmedien spielen sich dabei nicht selten gegenseitig die Bälle zu. Medien nehmen Einfluss darauf, welche Probleme in der Öffentlichkeit für relevant gehalten und letztlich Gegenstand politischen Handelns werden; sie schaffen das soziale Klima, in dem das Recht operieren muss. ${ }^{28}$ Die Auswirkungen einer derartigen Konstellation auf die Strafgesetzgebung, lassen sich am Gesetz zur Sicherungsverwahrung für nach Jugendstrafrecht Verurteilte exemplarisch beobachten.

\section{b) Verzicht auf Empirie}

Schon bei Schaffung des $\$ 66$ b StGB durch das Gesetz zur Einführung der nachträglichen Sicherungsverwahrung vom 23.7.2004 29 wurde eine fehlende Evaluation des Gesetzgebers moniert, welche die in der Gesetzesbegründung vorgebrachte Notwendigkeit der Regelung gestützt hätte ${ }^{30}$. Auch bei näherer Lektüre des vorliegenden Gesetzes zur Sicherungsverwahrung für Jugendliche bzw. dessen Entwurfs nebst Begründung sucht man eine solche Auswertung vergebens. Es fällt auf, dass stets die Rede ist von lediglich wenigen „Einzelfällen“, „Extremfällen“, „äußersten Ausnahmefällen“, bei denen die Voraussetzungen für eine Sicherheitsverwahrung überhaupt erfüllt sein werden. Eine tragfähige Erhebung, in welcher Größenordnung die nachträgliche Sicherungsverwahrung für Jugendliche oder Heranwachsende voraussichtlich zur Anwendung gelangen soll, hat der Gesetzgeber indes nicht vorgelegt. Ebenso fehlt jede Bezugnahme auf Kriminalitätsstatistiken, aus denen sich die Notwendigkeit der gesetzlichen Regelung ableiten ließe.

Zwar zählt die Pressemitteilung des Bundesministeriums der Justiz vom 20.6.2008 zum verabschiedeten Gesetz eine Reihe von Konstellationen, welche die Anordnung der Sicherungsverwahrung nunmehr gebieten könnten, exemplarisch auf. Sie schließt allerdings mit dem überraschenden Hinweis, es handele sich bei den Beispielen lediglich um fiktive Fälle ohne realen Hintergrund; die meisten der in den letzten Jahren bekannt gewordenen Sachverhalte hätten Erwachsene bzw. Verurteilungen nach allgemeinem Strafrecht betroffen. Festgestellt werden muss damit, dass der Gesetzgeber einen empirischen Nachweis der Erforderlichkeit einer solchen Gesetzesänderung schuldig geblieben ist.
Der Bedarf an wissenschaftlich gesicherten Aussagen über Kriminalität sowie Nachweise über die präventive Wirkung von Kriminalpolitik und Gesetzgebung werden mit der Verlagerung hin zu einer expressiven, an vermeintlichen Sicherheitsbedürfnissen orientierten Kriminalpolitik geringer. ${ }^{31}$ Der Erfolg derlei gesetzgeberischer Aktivitäten beurteilt sich dann vordergründig nur noch nach „Maßgabe der gefühlten Bedrohung, die in hohem Maße ... Medienkampagnen" steuern. ${ }^{32}$

\section{c) Schnelle Lösungen sind gefragt}

Der stete Rekurs auf außergewöhnlich seltene Ausnahmefälle steht in auffälligem Missverhältnis zur medienwirksamen Inszenierung des Themas in der politischen Auseinandersetzung. So war bereits die Debatte um die Ausweitung des Anwendungsbereichs der Sicherungsverwahrung für Erwachsene von martialischen Rhetoriken begleitet. Beispielsweise meldete sich kurz vor der parlamentarischen Sommerpause 2001 der damalige Bundeskanzler Schröder in einem Boulevard-Blatt zu Wort, wo er im Umgang mit Sexualstraftätern forderte: „...Wegschließen - und zwar für immer...“. In einem mit großem Medieninteresse verfolgten Fall verlangte er die „ganze Härte des Gesetzes“ für den zu diesem Zeitpunkt noch gar nicht ermittelten Täter, für welchen es „nur die Höchststrafe“ geben könne.

Warf die damalige CDU/CSU-Opposition dem Kanzler dabei noch eine Mischung aus Populismus und Unkenntnis vor, griff sie später die Bemerkung anlässlich eines Sexualmordes an einem neunjährigen Jungen in München wieder auf. Sie bemängelte jetzt diametral zum früheren Standpunkt, es sei bei den kämpferischen Ankündigungen des Bundeskanzlers geblieben. Die Bundesregierung gehöre zum „Kartell der Schuldigen“, da sie sich effektiven Maßnahmen gegen Kapitalverbrechen verweigere. ${ }^{33}$ Diese Tat in München bildete letztlich den Anlass für die bayerische Staatsregierung, die Sicherungsverwahrung ebenso für Jugendliche zu fordern, denn exakt dieser Täter hatte bereits elf Jahre zuvor einen Jungen getötet und war seinerzeit nach Jugendstrafrecht verurteilt worden. In der Haft gestand er den sexuellen Missbrauch eines weiteren Kindes. Nach Verbüßung einer Jugendhaftstrafe von mehr als neun Jahren wurde er entlassen, bevor er zehn Monate später die erneute Mordtat beging.

Die Jugendkriminalität stellt einen medialen Dauerbrenner dar: So beherrschte das Thema Jugendgewalt und Jugendstrafrecht zuletzt Anfang 2008 etwa den Landtagswahlkampf in Hessen. Auch damals reagierte die Politik auf Medienberichte über einen Vorfall in München, bei dem Jugendliche einen Rentner niedergeschlagen hatten. Zwar präsentierte man rasch einen Maßnahmenkatalog zur Verschärfung des Jugendstrafrechts; tatsächlich konnte jedoch der behauptete Anstieg der Jugendkriminalität, insbesondere der Gewaltkriminalität empirisch nicht belegt werden.

Der Gesetzgeber begnügt sich in der Entwurfsbegründung zum vorliegenden Gesetz gleichermaßen mit Bezugnahmen auf nicht näher bezeichnete „Beispiele der jüngsten Vergangenheit“. Zum Nachweis der Erforderlichkeit der vorgelegten Gesetzesänderung bemüht er den grundgesetzlichen Schutzauftrag des Staates gegenüber potentiellen Opfern und deren Rechten auf Leben und körperliche Unversehrtheit, welche durch erwartbare schwerwiegende Straftaten bedroht werden. Eine Auseinandersetzung mit Alternativen zur vorgelegten Gesetzesänderung findet nicht statt - unter Alternativen heißt es schlicht: „Keine“. 34

In ihrem Sicherheitsempfinden durch breite Medienberichterstattung über spektakuläre Verbrechen gestört, sucht die Bevölkerung 
nach Antworten, die verständlich sind und eine unmittelbar wirksame Bewältigung des Problems versprechen. Das geschwächte öffentliche Vertrauen in die Experten bildet den Nährboden für eine populistische Kriminalpolitik. Mit dem vorliegenden Gesetz hat die Legislative solch eine (vor-)schnelle Lösung vorgelegt.

\section{Sicherungsverwahrung als Maßregel für nach Jugendstrafrecht Verurteilte}

Mit der Einführung der Sicherungsverwahrung auf dem Gebiet des Jugendstrafrechts gehen erhebliche rechtliche wie auch praktische Schwierigkeiten einher. Dabei sind zum einen die in $\$ 7$ Abs. 2 JGG selbst normierten Voraussetzungen nicht unproblematisch, zum anderen stellt die Neuregelung mit dem Vollzug der Maßregel befasste Behörden vor enorme Herausforderungen.

\section{Die Regelung des $\mathbb{} 7$ Abs. 2 JGG}

In vielerlei Hinsicht wirkt die mit $\ 7$ Abs. 2 JGG geschaffene Norm zur Anordnung der Sicherungsverwahrung für nach Jugendstrafrecht Verurteilte misslungen. Die Vorschrift weist zahlreiche Mängel auf, welche an deren Verfassungsmäßigkeit zweifeln lassen.

\section{a) Ausgestaltung als nachträgliche Maßregel}

Zunächst fällt die Ausgestaltung der Sicherungsverwahrung als nachträgliche, mithin nicht unmittelbar im Urteil selbst angeordnete oder vorbehaltene Maßregel auf. ${ }^{35}$ Offenbar glaubte der Gesetzgeber hierdurch dem jungen Rechtsbrecher die mit einem solchen Ausspruch einhergehenden, negativen psychischen Auswirkungen während des Jugendstrafvollzugs ersparen zu können. ${ }^{36}$ Diese Annahme trifft indes keineswegs zu, weil auch ohne die explizite Anordnung in der Urteilsformel das Risiko der nachträglichen Festsetzung wie ein „Damoklesschwert“37 über dem Verurteilten schwebt. Ob sich eine solche latente Belastung förderlich auf den erzieherisch auszugestaltenden Vollzug der verhängten Jugendstrafe auswirkt, mag bezweifelt werden. Vielmehr lässt dies eine scheinbare bzw. vorgetäuschte Anpassung der Inhaftierten an den Jugendstrafvollzug erwarten, welche die Einsicht in begangenes Unrecht sowie die Erkenntnis von Fehlverhalten verhindert ${ }^{38}$ und sich damit diametral zum jugendstrafvollzuglichen Erziehungsziel gem. $\$ 2$ Abs. 1 S. 2 JGG verhält.

Tragendes Argument des Gesetzgebers für die Ausgestaltung als nachträgliche Maßregel bildet allerdings die Unmöglichkeit, die notwendige, hinreichend sichere Prognose zum Zeitpunkt der Verhängung des Strafurteils zu treffen. Da die Entwicklung der nach Jugendstrafrecht zu verurteilenden Personen regelmäßig noch nicht abgeschlossen ist, bleibt eine Feststellung der von $\$ 7$ Abs. 2 JGG vorausgesetzten Erwartung erneuter Straftaten der in der Norm bezeichneten Art zum Stichtag der Verurteilung ausgeschlossen. ${ }^{39}$ $\mathrm{Ob}$ aber eine solche Abschätzung mit der nötigen Gewissheit zu einem späteren Zeitpunkt während des Vollzugs getroffen werden kann, steht damit noch nicht fest. Eine künftige Gefährlichkeit jugendlicher oder reifeverzögerter Heranwachsender entzieht sich für gewöhnlich jeder Prognose, da die Umstände, welche den weiteren Werdegang eines jungen Menschen nachhaltig beeinflussen, vielfältig und vor allem nicht vorhersehbar sind. ${ }^{40}$ Eine realistische, für die Anordnung der Sicherungsverwahrung erforderliche Vorhersage über die Wahrscheinlichkeit der künftigen Begehung weiterer Straftaten schließt sich damit letztlich aus. ${ }^{41}$

Sofern man - anders als dies hier vertreten wird - mit dem Gesetzgeber der gewagten These folgt, auch bei jungen Rechtsbrechern lasse sich theoretisch eine entsprechende Gefährlichkeitsprognose treffen, so bleiben dennoch Zweifel. Denn gerade bezogen auf nach Jugendstrafrecht Verurteilte erfordert die Erstellung einer solchen Voraussage die Beachtung der vom Bundesverfassungsgericht aufgestellten besonderen Qualitätserfordernisse mit höchster Sorgfalt. ${ }^{42}$ Es fehlt indes bei Jugendlichen jeder Maßstab sowie jeder sonstige greifbare Anhaltspunkt, der eine Grundlage bei der Prognoseerstellung bieten könnte. Dies zu leisten obliegt nun wiederum den Gerichten, denen der Gesetzgeber mit $\mathbb{} 7$ Abs. 2 JGG eine faktisch nicht zu bewältigende Aufgabe aufbürdet.

\section{b) In der Vorschrift normierte Voraussetzungen}

Aber auch gegen die Voraussetzungen der Vorschrift im Übrigen bestehen gravierende Bedenken. So fragt sich weiter, zu welchem Zeitpunkt während des Vollzugs eine Anordnung der Sicherungsverwahrung stattfinden soll. Der Gesetzgeber ging offenbar davon aus, dies dürfe erst gegen Ende der Haftverbüßung erfolgen. ${ }^{43}$ Dass der Wortlaut des $\mathbb{7}$ Abs. 2 JGG eine solche Einschränkung jedoch nicht trägt, bedingt einen weiteren Unsicherheitsfaktor für den Betroffenen, der somit theoretisch zu jedem Zeitpunkt während seiner Inhaftierung mit einer Anordnung zu rechnen hat. Denn die Norm spricht lediglich von der Möglichkeit, die Sicherungsverwahrung nachträglich anzuordnen; die Festlegung auf einen bestimmten Zeitpunkt vor Haftende hingegen hat keinen Niederschlag im Gesetzeswortlaut gefunden.

Darüber hinaus knüpft $\ 7$ Abs. 2 JGG nicht an „neue“ Tatsachen an, wie dies etwa $\$ 66$ b Abs. 2 StGB vorsieht, welcher das Vorbild der neuen Regelung stellte. Stattdessen genügt es, wenn die fraglichen Umstände „vor Ende des Vollzugs...erkennbar sind“, was insoweit bereits zum Zeitpunkt des Urteils feststellbare bzw. festgestellte Tatsachen einschließt. ${ }^{44}$ Diese Gestaltung scheint zunächst Einwänden im Hinblick auf Art. 103 Abs. 3 GG zu begegnen. ${ }^{45}$ Was bereits bei Einführung des $\$ 66 \mathrm{~b}$ StGB heftig kritisiert wurde ${ }^{46}$ gilt umso mehr, wenn die Umstände, auf welche eine verlängerte Freiheitsentziehung gestützt wird, nicht erst nach Erlass des Urteils auftreten, sondern zu diesem Zeitpunkt bereits vorgelegen haben. Insoweit besteht das begründete Risiko einer doppelten Berücksichtigung sowohl bei Festlegung der Strafdauer wie auch bei der späteren Entscheidung über die Sicherungsverwahrung. Da es sich allerdings bei der Sicherungsverwahrung um eine Maßregel der Besserung und Sicherung handelt, welcher nicht der Charakter einer Strafe für begangenes Unrecht zukommt ${ }^{47}$, greift Art. 103 Abs. 3 GG in diesem Zusammenhang nicht $e^{4}{ }^{48}$, selbst wenn dies für den Betroffenen faktisch so wirken mag.

Überdies scheint aber eine solche Konstruktion die Voraussetzungen von $\ 66 \mathrm{StGB}$ und $\ 66 \mathrm{~b}$ Abs. 2 StGB sinnwidrig zu vermengen. Ebenso wie $\$ 66$ b (Abs. 2) $\mathrm{StGB}^{49}$ darf auch der neue $\mathbb{} 7$ Abs. 2 JGG nicht als Korrekturmittel fehlerhafter Entscheidungen instrumentalisiert werden. Zwar erscheint dies bezogen auf die angeordnete Strafdauer eher weniger wahrscheinlich, weil insoweit mit der Höchststrafe von zehn Jahren gem. $\$ 18$ Abs. 1 S. 1 JGG bei von $\$ 7$ Abs. 2 JGG mindestens vorausgesetzten sieben Jahren Jugendstrafe eher geringere Kapazität für eine nachträgliche Abänderung zu Lasten des Verurteilten bleibt. Bezieht man allerdings die Entscheidung über die Frage der Anwendung von Jugend- oder Erwachsenenstrafrecht in die Betrachtung mit ein, so ergibt dies einen deutlich umfangreicheren Spielraum für derartige Modifikationen.

Eine angeblich strengere Fassung der Norm lassen die darin geregelten Voraussetzungen ebenfalls nur auf den ersten Blick erkennen ${ }^{50}$ : 
(1) Die bei sieben Jahren festgesetzte Mindeststrafgrenze macht den Eindruck, willkürlich gewählt zu sein und wirkt keineswegs aus Verhältnismäßigkeitserwägungen heraus geboten. ${ }^{51}$ Zwar ist dem Gesetzgeber zuzugeben, dass sich die Verhängung einer freiheitsentziehenden Maßregel bei Jugendstrafen von nur sehr kurzer Dauer als unverhältnismäßig darstellen würde - warum eine Grenzziehung aber gerade bei sieben Jahren erfolgen musste, leuchtet nicht ein. Denn insoweit erschließt sich nicht, weshalb gegenüber der Regelung des $\$ 66$ b Abs. 2 StGB gerade eine Anhebung um zwei Jahre notwendig gewesen sein soll. Auch erscheint das Argument des Gesetzgebers, die Höchststrafe von zehn Jahren sei als maßgebliche Grenze nicht in Betracht gekommen, da sie in der Praxis viel zu selten verhängt werde ${ }^{52}$, unschlüssig, bezweckt man doch mit der (nachträglichen) Sicherungsverwahrung gerade jene wenigen Fälle besonders gravierender Kriminalität zu erfassen. Davon ausgehend, dass die Strafdauer anders als die Verhängung der Maßregel nicht an Gefährlichkeits- sondern an Schuld- und Erziehungsaspekten orientiert festgesetzt wird, wäre zumindest eine Annäherung an die Höchststrafe wünschenswert gewesen.

Zudem geht mit der Verknüpfung von Strafdauer und Zulässigkeit der Sicherungsverwahrung unweigerlich eine Verbindung der zu ersterer getroffenen Erwägungen mit der Anordnung der Maßregel einher. Insofern wird damit bereits zum Zeitpunkt des Urteilserlasses indirekt jedenfalls über die Möglichkeit einer späteren Verhängung der Sicherungsverwahrung eine abschließende Entscheidung getroffen. Gerade dies sollte aber insbesondere für Jugendliche und Heranwachsende durch die Ausgestaltung der Maßregel als nachträgliche vermieden werden.

Hinzu kommt, dass im Jugendstrafrecht - anders als vom Gesetzgeber in der Entwurfsbegründung vorausgesetzt ${ }^{53}$ - auch hohe Haftstrafen nicht zwingend allein nach Schuldgesichtspunkten verhängt werden, sondern sich deren Dauer vorwiegend nach erzieherischen Belangen richtet. Die Strafdauer bildet daher ein ungeeignetes Kriterium für die Entscheidung über die Zulässigkeit der Sicherungsverwahrung; selbst eine rein an Schuldaspekten orientierten Strafdauer unterstellt, kann deren Aussagekraft für die Notwendigkeit der Verhängung einer schuldindifferenten Maßregel der Besserung und Sicherung ${ }^{54}$ nicht erheblich sein.

(2) Ebenso bleibt fraglich, ob eine Beschränkung auf schwerste Straftaten ohne eine enge Begrenzung des Katalogs auf einige wenige, vor allem aber konkret bezeichnete Tatbestände erreicht werden kann. ${ }^{55}$ Zwar schränkt $\$ 7$ Abs. 2 JGG seinem hier wörtlich zu nehmenden Normtext $^{56}$ nach den Anwendungsbereich immerhin auf Verbrechen i. S. d. $\ 12$ Abs. 1 StGB gegen das Leben, die körperliche Unversehrtheit oder die sexuelle Selbstbestimmung sowie Taten nach \$2 251 StGB (ggf. i. V. m. \$\$ 252, 255 StGB) ein. Leichtere Vergehen, z. B. gem. $\$ 223$ Abs. 1 StGB, bleiben daher vom Anwendungsbereich ausgeschlossen und eröffnen nicht die Möglichkeit einer nachträglichen Anordnung der Sicherungsverwahrung. Jedoch werden derartige Taten realiter ohnehin kaum jemals zur Verhängung von Haftstrafen mit einer Dauer von mindestens sieben Jahren führen. Der Wortlaut entbehrt insoweit jeder weiteren Beschränkung auf einen bestimmten Straftatenkatalog, sondern bleibt in dieser Hinsicht $\mathrm{zu}$ allgemein gehalten und daher viel zu unpräzise. Insgesamt betrachtet lassen sich damit gegen die Bestimmtheit bzw. hinreichende Begrenzung der Norm dieselben Bedenken anführen wie gegen $\ 66 \mathrm{~b}$ Abs. 2 StGB. ${ }^{57}$ Vor allem aber vernachlässigt der Gesetzgeber durch die offene Formulierung der Vorschrift die Beachtung einer gewissen
Warnfunktion, welche geschlossenen Katalogen von Anlasstaten zukommt.

(3) $\mathrm{Zu}$ überzeugen vermag auch nicht die Formulierung des $\mathbb{\$} 7$ Abs. 2 JGG, welche eine Verurteilung des betreffenden Täters „wegen oder auch wegen..." Verbrechen der in der Norm bezeichneten Art vorsieht. Denn dies schließt eine Erstreckung auf Konstellationen ein, in denen gegen Jugendliche oder Heranwachsende eine nur zum Teil auf einer Tat i. S. d. $\$ 7$ Abs. 2 JGG beruhende Einheitsstrafe gem. $\$ 31$ JGG verhängt wird. ${ }^{58}$ Der Konstruktion lässt sich damit der Einwand entgegenhalten, die Anordnung nachträglicher Sicherungsverwahrung werde auf diese Weise von der Dauer einer verhängten Jugendstrafe abhängig gemacht, die zumindest teilweise gar keine Reaktion auf eine der genannten Anlasstaten bildet. Zudem benachteiligt diese Formulierung nach Jugendstrafrecht Verurteilte gegenüber Tätern, gegen die nach $\ 66$ b Abs. 2 StGB die Anordnung nachträglicher Sicherungsverwahrung möglich ist. Denn für diese wird vorausgesetzt, dass bei Wegfall der Einzelstrafen für Nicht-Katalogtaten die Gesamtstrafe dennoch nicht unter fünf Jahren gelegen hätte. ${ }^{59}$ Offenbar erkannte auch der Gesetzgeber dieses Problem, überging es jedoch mit dem bloßen Hinweis auf das Fehlen von Alternativen in Zusammenhang mit $\$ 31$ JGG. ${ }^{60}$ Die Frage einer notwendig extensiven Auslegung der Vorschrift vergleichbar der Problematik um $\$ 66$ Abs. 3 S. 2 StGB ${ }^{61}$ stellt sich bei der gewählten weiten Formulierung der Norm jedenfalls nicht.

\section{c) Unklare Prognosebasis}

Im Unklaren bleibt nach der aktuellen Fassung des $\ 7$ Abs. 2 JGG sowohl Rechtsanwender wie Betroffener über die Basis der Prognoseerstellung. So lässt der Wortlaut der Vorschrift aufgrund der Einbeziehung von Tatsachen, die bereits vor Erlass des Urteils erkennbar waren, deren Berücksichtigung zu, schließt aber gleichermaßen jene Umstände ein, die erst während des Vollzugs der Jugendstrafe zu Tage treten. Im Ansatz erscheint damit zunächst die Bewertungsgrundlage bei der Fertigung einer hinreichend sicheren Prognose verbreitert. $^{62}$

Fraglich bleibt aber beim jungen Rechtsbrecher, in welchem Verhältnis beide Aspekte zu gewichten sind. Schließt die noch nicht beendete Entwicklung des Jugendlichen bzw. Heranwachsenden zum Zeitpunkt des Urteilserlasses eine sichere Vorhersage über zu erwartendes Fehlverhalten sowie künftige Gefährlichkeit aus, so vermag gerade der Freiheitsentzug mit seinen negativen Konsequenzen die Biographie des jungen Verurteilten noch nachhaltig zu beeinflussen. Aus diesem Grund gestaltet sich die Berücksichtigung von Umständen, welche während des Jugendstrafvollzugs auftreten, als in höchstem Maße problematisch. Eine Aussage darüber, ob diese (Anpassungs-)Reaktionen auf die Inhaftierung oder Folge einer anhaltenden Gefährlichkeit des Jugendstrafgefangenen darstellen, kann zum Zeitpunkt des Haftendes damit nicht mehr rückwirkend getroffen werden.

Aber auch vor der Verurteilung liegende Umstände besitzen aufgrund des bloß punktuell einsehbaren Entwicklungsstandes eines jungen Menschen zu diesem Zeitpunkt nur eine äußerst geringe - nahezu überhaupt keine - Aussagekraft im Bezug auf die Gefährlichkeit des Betroffenen bei Haftende. Erweitert wurde somit lediglich der betrachtete Zeitraum, ohne dass damit eine Verbreiterung der Tatsachengrundlage als solcher einherging. Die vordergründige Ausdehnung der zur Beurteilung heranziehbaren Phase vermag im Ergebnis nicht über die nach wie vor fehlende Prognosebasis hinwegzuhelfen. 


\section{Umsetzung des $\mathbb{} 7$ Abs. 2 JGG in der Praxis}

Mit der Realisierung der neuen, in $\ 7$ Abs. 2 JGG normierten Sanktion gehen erhebliche praktische Probleme einher. Nach der Föderalismusreform bildet der Vollzug der Sicherungsverwahrung an nach Jugendstrafrecht Verurteilten eine Ländersache. Aus diesem Grund trifft das Gesetz zur Einführung der nachträglichen Sicherungsverwahrung bei Verurteilten nach Jugendstrafrecht hierzu keinerlei Regelung. Die Herausforderung, vor welcher die Länder nunmehr stehen, bleibt dabei nicht nur finanzieller Natur.

Völlig unrealistisch ist zunächst die Einschätzung des Gesetzgebers in der Gesetzesbegründung, welche den finanziellen Mehraufwand in diesem Zusammenhang als „eher gering“ einstuft. ${ }^{63}$ Zwar mag zutreffend sein, dass es in der Praxis lediglich wenige derartige Fälle geben wird; den damit einhergehenden Kostenaufwand verkleinert dieser Umstand jedoch keineswegs. Denn weil fundamentale Prinzipien des Strafvollzugs ebenso für in Sicherungsverwahrung befindliche Jugendliche und Heranwachsende Geltung beanspruchen, bleibt der Trennungsgrundsatz ${ }^{64}$ auch - und besonders - für diese zu beachten. ${ }^{65}$

So stellt sich bereits der gegenwärtige Zustand im Rahmen des Vollzugs der Sicherungsverwahrung an Erwachsenen als aus rechtsstaatlicher Sicht in hohem Maße bedenklich dar. Es werden hier die Sicherungsverwahrten, weil deren geringe Anzahl die Schaffung eigener Anstalten aus organisatorischen Gründen kaum zulässt, beispielsweise in Justizvollzugsanstalten untergebracht, dort allerdings (freilich nur soweit möglich) in separaten Abteilungen. ${ }^{66}$ Im Hinblick auf den Trennungsgrundsatz begegnet diese Handhabung in der Praxis größten Vorbehalten.

Verschärft wird die Situation bezogen auf Jugendliche und Heranwachsende zudem, weil diese als Sicherungsverwahrte nicht nur von den erwachsenen Inhaftierten getrennt untergebracht, sondern insoweit gleichermaßen von den übrigen nach Jugendstrafrecht Verurteilten separiert werden müssen. Da aber bereits die Einhaltung des Grundsatzes der Trennung Jugendstrafgefanger von im Vollzug der Freiheitsstrafe befindlichen Inhaftierten vielerorts nicht gewährleistet ist ${ }^{67}$, bleibt die Verwirklichung der getrennten Unterbringung sicherungsverwahrter Jugendlicher und Heranwachsender von den anderen Jugendstrafgefangenen kaum zu erwarten. Für weibliche Jugendstrafgefangene verschärft sich diese Problematik wegen der noch geringeren Inhaftiertenzahlen in gesteigertem Maß. Diese Lage wird auch nicht durch das Argument entschärft, Jugendliche und Heranwachsende würden ohnehin mit Vollendung des 18. Lebensjahres vom Jugendstrafvollzug ausgenommen, sofern sie sich dafür nicht mehr eignen. Denn weil eine Herausnahme erst mit vollendetem 24. Lebensjahr erfolgen soll, $\mathbb{S} 91$ Abs. 1 S. 2 JGG, bleibt rechnerisch bei einer angenommenen Verurteilung im Alter von 14 Jahren zu einer siebenjährigen Jugendstrafe für die Dauer von drei Jahren (von vollendetem 21. bis vollendetem 24. Lebensjahr) die Vollstreckung der Sicherungsverwahrung an einem regulär im Jugendstrafvollzug zu Inhaftierenden denkbar.

Insofern macht die Schaffung des $\mathbb{} 7$ Abs. 2 JGG gleich in mehrfacher Hinsicht organisatorische Umstrukturierungen erforderlich, welche unweigerlich mit einem nicht zu unterschätzenden finanziellen Aufwand verbunden sein werden. Eine Realisierung des Vollzugs der Sicherungsverwahrung an jungen Verurteilten, welche den gesetzlichen Erfordernissen vollumfänglich entspricht, erscheint damit bereits aufgrund der geringen zu erwartenden Fallzahlen sowie den entstehenden Kosten ausgeschlossen. Dass der Bundesgesetzgeber hier kein Problem erkennen konnte, verwundert nicht, fällt doch der Vollzug der Sicherungsverwahrung in den Kompetenzbereich der Länder. An diesen bleibt es nun, die gerade erst neu geschaffenen Jugendstrafvollzugsgesetze um die erforderlichen Regelungen zur Sicherungsverwahrung an Jugendlichen und nach Jugendstrafrecht verurteilten Heranwachsenden zu ergänzen und diese entsprechend umzusetzen.

\section{Generelle Bedenken gegen eine Sicherungsverwahrung für Jugendliche}

Kontraproduktiv wirkt die Sicherungsverwahrung - insbesondere bei nachträglicher Anordnung - da längerfristig betrachtet bei den meisten Jugendlichen die Episode abweichenden bzw. kriminellen Verhaltens im Alter zwischen 20 und 25 Jahren endet. ${ }^{68}$ Insoweit vermag jedoch eine verlängerte Unterbringung in der Sicherungsverwahrung aufgrund der mit dem andauernden Freiheitsentzug einhergehenden, negativen Prägung eines jungen Menschen gerade das Eintreten dieses natürlichen Remissionseffekts zu verzögern oder gar gänzlich zu verhindern.

Die Annahme, Jugendkriminalität sei im Steigen begriffen und erfordere daher verschärfte Reaktionen, geht ebenfalls fehl. Seit 2001 befinden sich sowohl die Tatverdächtigenzahlen der polizeilichen Kriminalstatistik wie auch die Anzahl der Verurteilten bezogen auf Jugendliche und Heranwachsende im Sinken. ${ }^{69}$ Allgemein vermag man damit keinen statistisch belegbaren Anlass zu mehr Härte in diesem Bereich festzustellen.

Überdies besteht kein Konnex zwischen verschärften strafrechtlichen Reaktionen und gesteigerter Effizienz im Sinne einer geringeren Rückfälligkeit. Denn Täter, deren Verstöße mit ambulanten Sanktionen geahndet wurden, tendierten erheblich seltener zu erneuter Straffälligkeit als diejenigen Jugendlichen und Heranwachsenden, deren Verfehlungen mit Jugendstrafe oder Arrest begegnet wurde. ${ }^{70}$ Selbst Jugendstrafen, deren Vollstreckung zur Bewährung ausgesetzt wurde, scheinen weniger oft eine Rückfälligkeit zu bedingen als beispielsweise ein vollstreckter Arrest. ${ }^{71}$

Auch im europaweiten Vergleich bildete das deutsche Jugendstrafrecht in seiner Ausgestaltung vor in Kraft treten des Gesetzes zur Einführung der nachträglichen Sicherungsverwahrung bei Verurteilungen nach Jugendstrafrecht keine Ausnahme. Kennen die Niederlande und England auch vergleichbare Sanktionen, so bedürfen diese stets einer Anordnung im Strafurteil selbst - deren nachträgliche Verhängung bleibt in jedem Fall ausgeschlossen. ${ }^{72}$ Zudem fassen die ausländischen Rechtsordnungen die Sicherungsverwahrung primär als ein Mittel der Erziehungsförderung, nicht aber als eine Maßnahme zum Schutz der Allgemeinheit vor künftigen Straftaten auf. ${ }^{73}$

\section{Ausblick}

Den aufgezeigten Bedenken gegen die Sicherungsverwahrung für nach Jugendstrafrecht Verurteilte kommt beträchtliches Gewicht zu. Insgesamt erweckt die gesetzliche Neuerung den Anschein, mehr ein Mittel zur Demonstration von Entschlossenheit und Handlungsstärke denn eine notwendige Reaktion auf die tatsächlichen Verhältnisse zu sein. Mittels derartiger Kunstgriffe können Regierungen vorübergehend Ängste in der Bevölkerung sedieren und über die eigene Ratlosigkeit hinwegtäuschen. ${ }^{74}$

Bedenklich wirkt hierbei insbesondere, dass die durch mediale Suggestion erzeugte bzw. überspitzt dargestellte Bedrohung auf gleichem 
Weg auch wieder eliminiert werden kann. Insofern dürften mögliche künftige, scheinbar positive Effekte der Neuregelung wohl eher auf das Fehlen einer entsprechenden Inszenierung der Thematik - wie in der jüngsten Vergangenheit geschehen - zurückzuführen sein. Freilich bleibt jeder Erfolg der Gesetzesänderung ein illusorischer, wenn bereits das zu beseitigende Problem größer dargestellt wird, als es in Wahrheit ist. Nicht leugnen lässt sich das Verbleiben eines gewissen Restrisikos durch gefährliche Täter - ein solches vermag allerdings auch die Einführung der nachträglichen Sicherungsverwahrung nach Jugendstrafrecht Verurteilte aus den o. g. Gründen ${ }^{75}$ nicht zu beseitigen. Notwendig und ehrlicher wäre eine öffentliche Debatte über die Frage, in welchem Maß die Gesellschaft ein derartiges (Rest-)Risiko aushalten muss. Dies scheut jedoch nicht nur der Gesetzgeber.

\section{Die Autoren sind wiss. Ass. am kriminologischen Lehrstubl der Uni- versität Würzburg.}

\section{Fußnoten:}

Dr. Nina Nestler ist wissenschaftliche Assistentin am Lehrstuhl für Kriminologie und Strafrecht der Universität Würzburg; Christian Wolf ist wissenschaftlicher Mitarbeiter am Lehrstuhl für Strafrecht, Strafprozessrecht, Rechtstheorie, Informationsrecht und Rechtsinformatik an derselben Universität.

1 BGBl I, S. 1212 f.

2 Ähnlich Laubenthal, Die Renaissance der Sicherungsverwahrung, ZStW 2004, S. 706 f.; zum strafrechtlichen Charakter des Maßregelrechts Gazeas, Nachträgliche Sicherungsverwahrung - Ein Irrweg der Gesetzgebung?, StraFo 2005, S. 13; Pieroth, Gesetzgebungskompetenz und Grundrechtsfragen der nachträglichen Sicherungsverwahrung, JZ 2002, S. 926.

3 So handelt es sich etwa bei Beispielen, welche in der Pressemitteilung des Bundesministeriums der Justiz vom 20.06.2008 zur Sicherungsverwahrung nach Jugendstrafrecht angeführt werden, lediglich um erdachte und damit fiktive Fälle; dazu auch Eisenberg, Nachträgliche Sicherungsverwahrung bei zur Tatzeit Jugendlichen bzw. Heranwachsenden?, JZ 2007, S. 1143.

4 Laubenthal (Fn. 2), S. 703 f.; Ostendorf, Jugendstrafrecht - Reform statt Abkehr, StV 2008, S. 149.

5 Von Liszt, Der Zweckgedanke im Strafrecht, in: ders. (Hrsg.), Strafrechtliche Aufsätze und Vorträge, 2 Bd., Berlin 1905, S. 126, 169

6 RGBI I, S. 995

7 BGBl I, S. 717.

8 So Laubenthal, (Fn. 2), S. $703 \mathrm{ff}$.

9 Braum, Nachträgliche Sicherungsverwahrung: In dubio pro securitate? Wegsperren ohne tragfähige Legitimation, ZRP 2004, S. 105 ff.

10 BGBl I, S. 160.

11 BGBl I, S. 3344.

12 BVerfGE 109, S. 190 ff.

13 Poseck, Das Gesetz zur Einführung der nachträglichen Sicherungsverwahrung, NJW 2004, S. 2561, der das Gesetz aus kriminalpolitischer Sicht für „nicht durchweg zufrieden stellend“ hält.

14 Kinzig, Umfassender Schutz vor dem gefährlichen Straftäter? - Das Gesetz zur Einführung der nachträglichen Sicherungsverwahrung NStZ 2004, S. 655,658

15 Siehe dazu unten Abschn. III. 1. c).

16 BGH, NJW 1975, S. 1666

17 BGH, NStZ-RR 2001, S. 13; siehe dazu unten Abschn. III. 1. a).

18 BGBl I, 2003, S. 3007.

19 BGBl I, 2004, S. 1838.

20 BT-Drs. $15 / 2887$, S. 18.

21 BVerfGE 109, S. 133 ff.; BVerfG NJW 2004, S. 750 ff.

22 Vgl. Gesetzentwurf des Bundesrates, BR-Drs. 238/04 v. 14.5.2004.

23 BR-Drs. 238/04, S. 23.

24 BR-Drs. 138/06, S. 1

25 Braum, (Fn. 9), S. 105.

26 Düx, Sexualstraftaten und Sicherungsverwahrung - Abschied vom rechtstaatlichen Strafverfahren?, ZRP 2006, S. 82, 83.

27 Bauman, Die Krise der Politik - Fluch und Chance einer neuen Öffentlichkeit, Hamburg 2000, S. 81

28 Held, Medienkultur und Demokratie, in: Brunkhorst (Hrsg.), Demokratischer Experimentalismus. Politik in der komplexen Gesellschaft, Frankfurt a. M. 1998, S. 73.

29 BGBl I, S. 1838.

30 Kinzig (Fn. 14), S. 655 f.

31 Albrecht, Öffentliche Meinung, Kriminalpolitik und Kriminaljustiz, in: Walter/Kania/Albrecht (Hrsg.), Alltagsvorstellungen von Kriminalität, Individuelle und gesellschaftliche Bedeutung von Kriminalitätsbildern für die Lebensgestaltung, Münster 2004, S. 496.
32 Fischer, Strafgesetzbuch, 55. Aufl., München 2008, \$66b, Rn. 4

33 Der damalige CSU-Generalsekretär Markus Söder äußerte sich seinerseits via BILD am Sonntag: „Der Kanzler hat einst getönt, Kindermörder für immer wegzusperren - passiert ist gar nichts. Schuld hat zwar immer der Täter, bei Wiederholungstätern aber ist Schröder indirekt für jedes Verbrechen an Kindern mitverantwortlich - weil Rot-Grün keine einzige Regel verschärft hat.", vgl. dpa-Meldung in der Süddeutschen Zeitung, http:// www.sueddeutsche.de/,tt1m3/deutschland/artikel/535/48487/ (7.9.2006). Den Hintergrund bildete der Sexualmord einem neunjährigen Jungen aus München; der Täter war bereits wegen eines Kindermordes vorbestraft.

34 BT-Drs. 16/6562, S. 2.

35 Dazu BVerfG, StV 2006, S. 574; krit. Ostendorf (Fn. 4), S. 152.

36 Vgl. die Gesetzesbegründung, BT-Drs. 16/6562, S. 7.

37 Dazu schon im Rahmen des $\$ 106$ JGG Kreuzer, Nachträgliche Sicherungsverwahrung - rote Karte für gefährliche Gefangene oder für den rechtsstaatlichen Vertrauensschutz?, ZIS 2006, S. 151; Ostendorf (Fn. 4), S. 152; Ostendorf, Jugendgerichtsgesetz, 7. Aufl., Baden-Baden 2007, \$106 Rn. 12.

38 Ostendorf (Fn. 4), S. 152.

39 BT-Drs. 16/6562, S. 7; Eisenberg (Fn. 3), S. 1143 f.

40 Ostendorf (Fn. 4), S. 152; diese räumt auch die Gesetzesbegründung ein, vgl. BT-Drs. 16/6562, S. 7, 8, 9 .

41 In diesem Sinne auch Ostendorf, Nachträgliche Sicherungsverwahrung bei jungen Menschen auf dem internationalen und verfassungsrechtlichen Prüfstand, ZRP 2007, S. 148.

42 BT-Drs. $16 / 6562$, S. 7.

43 BT-Drs. $16 / 6562$, S. 7.

44 BT-Drs. 16/6562, S. 7

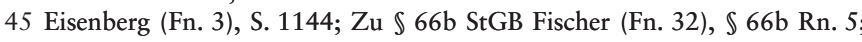
Gazeas (Fn. 2), S. 13.

46 Siehe dazu Fischer (Fn. 32), $\ 66$ b Rn. 5 m. zahlr. Nachw.

47 BVerfG, NJW 2004, S. 744 im Zshg. mit Art. 103 Abs. 2 GG

48 Jarass/Pieroth, Grundgesetz, 8. Aufl., München 2006, Art. 103 Rn. 59.

49 Dazu Detter, Zum Strafzumessungs- und Maßregelrecht, NStZ 2008, S. 272.

50 Anders jedoch die Pressemitteilung des Bundesministeriums der Justiz vom 20.06.2008 zur Sicherungsverwahrung nach Jugendstrafrecht.

51 So aber die Gesetzesbegründung BT-Drs. 16/6562, S. 9.

52 BT-Drs. 16/6562, S. 9

53 BT-Drs. 16/6562, S. 7.

54 Fischer (Fn. 32), vor $\$ 61$ Rn. 1.

55 Im Zshg. mit $\int 66$ Abs. 3 StGB Laubenthal (Fn. 2), S. 716

56 So bzgl. $\$ 66$ b Abs. 2 StGB Fischer (Fn. 32), \$ 66b Rn. 12

57 Dazu BVerfG, StV 2006, S. 574 ff.; krit. zu \$66b StGB Eisenberg, Nachträgliche Sicherungsverwahrung?, ZfStrVo 2001, S. 131; Gazeas (Fn. 2), S. 13; Jansing, Nachträgliche Sicherungsverwahrung, Entwicklungslinien in der Dogmatik der Sicherungsverwahrung, Münster 2004, S. 319-321; Kinzig, Das Gesetz zur Einführung der vorbehaltenen Sicherungsverwahrung, NJW 2002, S. 3206; Kinzig, Umfassender Schutz vor dem gefährlichen Straftäter? - Das Gesetz zur Einführung der nachträglichen Sicherungsverwahrung, NStZ 2004, S. 657; Ullenbruch, Nachträgliche Sicherungsverwahrung - ein legislativer „Spuk“ im judikativen „Fegefeuer“?, NStZ 2007, S. $62 \mathrm{ff}$.

58 Eisenberg (Fn. 3), S. 1144.

59 Siehe zur Berechnung der Freiheitsstrafe im Rahmen des $\$ 66 \mathrm{~b}$ StGB bei Verurteilung zu einer Gesamtstrafe Fischer (Fn. 32), \$66b Rn. 13; vgl. auch Kinzig (Fn. 57), S. 658.

60 BT-Drs. 16/6562, S. 9.

61 Dazu Laubenthal (Fn. 2), S. 721.

62 Vgl. auch Laubenthal (Fn. 2), S. 740.

63 BT-Drs. 16/6562, S. 2.

64 Laubenthal, Strafvollzugs, 5. Aufl., Heidelberg 2008, Rn. 58 f.

65 Eisenberg (Fn. 3), S. 1144; Laubenthal (Fn. 64), Rn. 874a f.

66 Ullenbruch (Fn. 57), S. 63.

67 Laubenthal (Fn. 2), S. 710; Schönberger, Zur justitiellen Handhabung der Voraussetzungen der Unterbringung gemäß $\$ \$ \$ 63,66$ StGB, Berlin 2002, S. 189 .

68 Ostendorf (Fn. 4), S. 152.

69 Vgl. dazu Ostendorf (Fn. 4), S. 150.

70 Ostendorf (Fn. 4), S. 150; Ostendorf, Gegen die Abschaffung des Jugendstrafrechts oder seiner Essentialia, NStZ 2006, S. 323 f.; Verrel, „Warnschussarrest“ - Kriminalpolitik wider besseres Wissen?, NStZ 2008, S. 178; zum Ganzen Jehle/Heinz/Sutterer, Legalbewährung nach strafrechtlichen Sanktionen, Berlin 2003.

71 Ostendorf (Fn. 4), S. 150.

72 Vgl. dazu Ostendorf (Fn. 41), S. 147, insbes. auch zur Vereinbarkeit mit internationalen Vorgaben.

73 Ostendorf (Fn. 41), S. 147

74 Wolf, Das Stigma als Mittel moderner Kriminalprävention, in: Hilgendorf (Hrsg.), Dimensionen des IT-Rechts, Berlin 2008, S. 135.

75 Vgl. oben Abschn. III. 